\title{
Quelques aspects de l'assurance \\ dans les relations économiques internationales: \\ l'Assurance-Crédit à l'exportation, l'Assurance-Cautionnement à l'étranger et l'Assurance des investissements privés à l'étranger
}

\author{
par Luciano Kramar *
}

\begin{abstract}
Dans le contexte du développement des relations économiques internationales, l'assurance (publique ou privée, directe ou indirecte) est appelée à jouer un rôle de plus en plus important, en tant que moyen susceptible de couvrir certains risques dont la réalisation, virtuelle ou effective, peut constituer un obstacle au développement des échanges de marchandises et de capitaux.
\end{abstract}

Ce texte constitue seulement une première tentative pour pouvoir mieux comprendre certains aspects de l'assurance et du rôle qu'elle peut jouer dans les relations économiques internationales.

Nous avons donc essayé de mettre en évidence les raisons qui expliquent le développement et l'importance de l'assurance et de procéder à une analyse descriptive et préliminaire de trois domaines spécifiques $\mathbf{1}$ :

- l'Assurance-Crédit à l'exportation (de biens et de services) ;

- l'Assurance-Cautionnement à l'étranger ;

- l'Assurance des investissements privés à l'étranger.

Ce travail nous a ensuite servi pour dégager quelques aspects qui pourraient faire l'objet de recherches plus approfondies et plus détaillées.

\section{L'Assurance-Crédit à l'exportation}

\subsection{Introduction}

L'Assurance-Crédit à l'exportation s'est développée à la fin du siècle dernier au Royaume-Uni ; elle a connu un développement lent, mais s'est toujours mise en vedette quand les circonstances économiques devenaient difficiles.

* Chercheur, chef de projet.

1 Ces trois domaines ont été choisis non seulement à cause de leur développement actuel, mais aussi parce que les opérations économiques qu'ils impliquent peuvent être liées (par exemple l'investissement privé à l'étranger intervenant à la suite du développement des exportations dans un pays donné). 
Ce fut le cas notamment après la première guerre mondiale ${ }^{2}$ et au cours des années $30^{3}$. Au début des années 50, avec la reprise économique de l'Europe occidentale et du Japon, la structure des échanges internationaux s'est transformée en raison du développement des exportations de biens d'équipement et la tendance du marché s'est renversée au profit des acheteurs (" buyer market 》).

Dans les pays industrialisés le crédit à l'exportation est progressivement devenu : - un moyen de favoriser l'exportation de biens et de services ${ }^{4}$;

- un instrument de la politique d'assistance aux pays en voie de développement 5.

$\mathrm{Au}$ cours de la présente décennie, les problèmes de balance des paiements ont encore accentué le recours aux crédits à l'exportation, qui sont considérés comme essentiels pour maintenir la compétitivité extérieure des industries nationales, soumises à une concurrence internationale de plus en plus intense.

\subsection{Le financement des exportations}

Dans toute exportation, deux intérêts divergents s'opposent : celui du vendeur, qui désire garder un titre légal sur les produits qu'il vend jusqu'à leur paiement, ou du moins jusqu'à ce qu'il ait l'assurance qu'il sera payé, et celui de l'acheteur, qui répugne à payer avant d'avoir reçu les marchandises.

On peut donc rencontrer deux cas «extrêmes» dans le financement des exportations :

- celui-ci est accordé par l'exportateur, qui en assure intégralement la charge (ex. paiement en compte courant, envoi en consignation);

- celui-ci est supporté par l'importateur (ex. paiement comptant).

Dans la pratique ces cas sont assez rares ; nous venons de voir qu'actuellement la tendance du marché était en faveur de l'acheteur : c'est donc le vendeur qui, directement ou indirectement, doit accorder un crédit. Il peut prendre deux formes:

- crédit-fournisseur :

il est accordé directement par l'exportateur à l'importateur. Le vendeur peut financer ce crédit en obtenant un prêt auprès d'une banque commerciale. Ces prêts pourront être réescomptés par l'établissement national de crédit à l'exportation ou par la Banque centrale;

2 Quand il s'agissait de combattre le chômage, qui était généralisé.

3 Où l'on voulait atténuer les effets de la dépression, en maintenant un courant d'exportation malgré la désorganisation des marchés, les crises de changes et l'instabilité politique.

4 Les crédits à l'exportation constituent de nos jours une des principales formes de subventions aux exportations dans les pays développés. Cette subvention résulte :

-- des facilités de financement ou refinancement à des taux inférieurs à ceux existant sur le marché ;

- de l'Assurance-Crédit à l'exportation offrant des garanties de plus en plus étendues.

5 Le recours excessif aux crédits à l'exportation, utilisés pour le financement des programmes de développement, a conduit à de nombreux problèmes pour le remboursement de la dette dans les pays en voie de développement. Selon le rapport Pearson, en 1968 les crédits à l'exportation ne constituaient que le quart de la dette extérieure de ces pays, mais ils absorbaient la moitié environ du service de la dette. 
- crédit-acheteur :

il est accordé directement à l'importateur, par un établissement de crédit du pays exportateur, pour lui permettre de payer l'exportateur au comptant. Le rôle de la Banque centrale, ou des établissements nationaux de crédit à l'exportation, est alors essentiel pour la mobilisation des crédits.

Que ce soit directement (par l'avance de fonds à des taux inférieurs à ceux du marché) ou indirectement (par le réescompte à des conditions particulièrement avantageuses), le dernier mot dans cette " course au crédit subventionné » revient aux autorités nationales ${ }^{6}$.

\subsection{Définition des risques}

En Assurance-Crédit on distingue deux catégories de risques :

- les risques commerciaux ;

- les risques non commerciaux, ou risques politiques.

Dans la première sont classés l'insolvabilité de l'importateur (prouvée ou prolongée) et le risque que l'acheteur refuse d'accepter les marchandises exportées, sans qu'il n'y ait aucune faute de l'exportateur.

Sous la définition de "risques politiques» on englobe notamment les circonstances suivantes :

- risque de guerre ou troubles, ou encore de catastrophes naturelles, dans le pays importateur ;

- risque de non-transfert : l'Etat, dont l'importateur est ressortissant, cesse de mettre des devises à la disposition des importateurs (faute de réserves de change, par exemple) ;

- risque d'annulation ou de non-renouvellement d'une licence d'importation ;

- risque d'application de nouvelles restrictions aux importations (ou exportations) qui n'existaient pas au moment de la conclusion du contrat ;

- risque de change si le contrat est rédigé en devises étrangères ${ }^{7}$;

6 Economiquement ces facilités s'analysent comme des subventions aux exportations : elles se traduisent par un transfert de ressources réelles des contribuables aux exportateurs et des consommateurs domestiques de biens exportables aux producteurs. Elles produisent donc des distorsions dans l'allocation des ressources entre les secteurs d'exportation et les secteurs concurrents.

7 Il y a trois types de garanties:

- Garantie de change sur rapatriements. Elle couvre la perte subie par l'exportateur en cas de baisse du cours de la monnaie dans laquelle est libellé un contrat d'exportation ;

- Garantie de change sur transfert de devises. Couvre la hausse du cours de la devise dans laquelle est libellé un contrat d'achat de fournitures étrangères nécessaires à l'exécution du contrat d'exportation ;

- Garantie de change sur caution. Couvre la banque ayant souscrit un engagement de caution pour garantir un exportateur en cas de hausse du cours de la devise dans laquelle est libellée cette caution. 
- risque d'inflation par les coûts dans le pays de l'exportateur ${ }^{8}$.

La plupart des systèmes d'assurance donnent à l'exportateur la possibilitée de couvrir les risques courus, aussi bien pendant la période de production avant l'expédition qu'après l'expédition. L'assurance pré-expédition, conçue spécialement pour les exportateurs qui produisent des marchandises selon les spécifications de l'importateur, couvre normalement le risque d'insolvabilité, de l'annulation de commande sans remboursement des frais encourus, ou encore d'événements politiques (ou catastrophes naturelles) qui mettent l'exportateur dans l'impossibilité de livrer les marchandises.

Nous pouvons mentionner enfin l'existence de polices d'Assurance-prospection (permettant de couvrir les frais d'étude de marché, les frais de voyage et de séjour, etc.) et $d$ 'Assurance-foire (garantissant une partie des fonds versés pour la location d'un stand, son aménagement, les frais d'expédition, etc., au cas où les prises de commandes seraient insuffisantes).

Pourtant, si la gamme de risques assurables par l'Assurance-Crédit est très large, ainsi que nous l'avons vu, ce système ne couvre pas les pertes qui auraient pu être assurées par un autre type de police (ex. Assurance maritime, Assurance-incendie), ni celles découlant de l'inexécution de la part de l'exportateur des clauses du contrat ${ }^{9}$.

\subsection{Rôle de l'Assurance-Crédit dans le financement des exportations}

L'Assurance-Crédit joue un rôle d'aide pour l'exportateur dans la recherche des fonds, en lui fournissant une garantie additionnelle. Le moyen le plus simple consiste à souscrire une police d'assurance et à en transférer le bénéfice à une banque. Mais du point de vue de cette dernière, la garantie n'est pas toujours suffisante, car :

- cette police pourrait être invalidée si l'exportateur ne respectait pas les stipulations du contrat ;

- cette police ne couvre généralement pas le paiement des intérêts qui s'accumuleraient entre les dates d'échéance des traités d'exportation impayés et les dates auxquelles les indemnités seront versées par la compagnie d'Assurance.

Ainsi certains systèmes d'Assurance prévoient l'octroi, à la banque qui finance l'exportation, d'une garantie directe et inconditionnelle, qui ne dépend pas de la validité du droit à l'indemnité que l'exportateur tient de sa police.

On constate clairement le lien étroit entre l'Assurance-Crédit et le financement des exportations. L'assurance :

- permet aux exportateurs de vendre à crédit sans que l'équilibre de leurs finances soit compromis par l'acceptation de risques excessifs;

- facilite la mobilisation des crédits à l'exportation lorsque l'exportateur ne peut pas financer lui-même ses transactions.

8 La Commission de Bruxelles a demandé aux pays qui offrent cette police (France, Royaume-Uni et Italie) de mettre un terme à cette activité, qui est contraire à l'article XVI du GATT, pour la fin de l'année 1978.

9 Il est vrai, pourtant, qu'au Royaume-Uni, par exemple, les banques qui financent les exportations peuvent recevoir une garantie inconditionnelle de $100 \%$ de leurs avances pour l'exportation, et se voient au surplus garantir un rendement certain, bien que limité. 


\subsection{Les sociétés qui couvrent les risques des crédits à l'exportation}

Nous avons vu à la section 1.3. qu'il y avait deux catégories de risque. A une exception près, que nous verrons plus loin, ces deux catégories sont assurées par deux systèmes différents, savoir :

- les risques commerciaux par les assurances privées ou gouvernementales et les sociétés d'affacturage ;

- les risques politiques par les assurances gouvernementales seulement.

Cette répartition reste valable quelles que soient, par ailleurs, les variantes utilisées dans chaque pays.

Nous allons voir maintenant les principales caractéristiques des divers systèmes d'assurance.

\section{ORGANISMES GOUVERNEMENTAUX}

Ces organismes existent dans la plupart des pays industrialisés et de plus en plus dans les pays en voie de développement, ainsi d'ailleurs que dans les pays à économie planifiée.

Généralement ils permettent d'assurer, contre les risques politiques et commerciaux :

- les biens et les services d'origine nationale;

- les biens ayant un pourcentage de matières premières et de travail d'origine nationale suffisant.

\section{COMPAGNIES D'ASSURANCE PRIVEES}

Ces sociétés garantissent normalement les crédits à l'exportation à court et à moyen terme contre les risques d'insolvabilité prouvée 10 ou prolongée. Elles utilisent le système de la réassurance pour limiter et répartir les risques $\mathbf{1 1}$.

Cette assurance est accordée selon les principes commerciaux, c'est-à-dire par la fixation de primes qui, en raison des opérations en cause, doivent permettre de :

- fournir un fonds de roulement suffisant ;

- constituer une réserve pour couvrir les pertes éventuelles ;

- constituer une réserve pour imprévu.

Les principes utilisés sont :

- la globalité, pour éviter une pré-sélection des risques;

- la participation de l'assuré à une partie des risques ;

- la limitation de la couverture accordée à un risque donné.

10 En effet les dangers de collusion entre les exportateurs et les importateurs sont importants.

11 C'est ainsi que l'on trouve, dans certains pays qui n'ont pratiquement pas de systèmes privés d'Assurance-Crédit des sociétés privées qui réassurent cette catégorie de risques auprès de compagnies étrangères. 
Ajoutons que la réglementation nationale impose souvent à ces sociétés de constituer des réserves de crise importantes, étant donné les particularités de cette activité 12 .

\section{L'AFF ACTURAGE}

Cette technique de financement à court terme des exportations (très ancienne puisqu'elle remonte au XIVe siècle) permet de décharger l'exportateur :

- du risque d'insolvabilité ;

- du recouvrement des créances à l'exportation ;

- de la gestion des créances;

tout en lui fournissant une avance de fonds. Bien que la majorité des opérations traitées par les sociétés d'affacturage relève de l'affacturage interne, nombre d'entre elles s'occupent également d'opérations internationales.

La plupart des sociétés d'affacturage appartiennent à des banques (ou à des sociétés d'assurance) ${ }^{13}$, dont elles sont tributaires pour leurs besoins de crédit.

Par rapport aux activités bancaires de financement des exportations, l'affacturage présente par contre les caractéristiques suivantes :

- l'affactureur acquiert les créances de l'exportateur sans possibilité de recours envers celui-ci ;

- l'affactureur est principalement concerné par la côte de crédit de l'importateur étranger ;

- l'affactureur fixe une limite de crédit, non pas pour l'exportateur mais pour chaque importateur ;

- l'affactureur doit veiller à une bonne répartition des risques entre divers produits et divers pays.

Les services offerts par cette technique sont les suivants :

- couverture du risque d'insolvabilité :

cette garantie couvre la totalité du risque de perte si le contrat n'est pas honoré pour cause d'insolvabilité de l'acheteur, mais uniquement pour cette cause ${ }^{14}$;

- recouvrement et comptabilité :

les factures établies par l'exportateur sont payables directement à l'affactureur, qui se charge également du recouvrement, si nécessaire ;

- avance de fonds:

normalement l'affactureur s'engage à payer la créance, dès que la marchandise est expédiée à l'importateur, ce qui permet à l'exportateur de mobiliser les créances

12 Il y a une exception à la situation que nous venons de décrire : elle est constituée par la société Investment Insurance International, du groupe Lloyds. Récemment, cette compagnie a entrepris une activité Assurance-Crédit à l'exportation et Assurance-cautionnement, permettant de couvrir certains risques non commerciaux.

13 Les sociétés d'affacturage peuvent se couvrir du risque d'insolvabilité qu'elles supportent vis-à-vis de leurs clients en utilisant les services de l'assurance ou de la réassurance.

14 Il est rare, en effet, que la garantie couvre le risque de change ou le risque de transfert. 
acquises avant leur échéance. Dans la pratique, c'est bien cette possibilité qui constitue une des causes majeures du succès de l'affacturage.

Il y a deux types de sociétés qui s'occupent d'affacturage à l'exportation :

- celles qui opèrent par l'intermédiaire de filiales (ex. International Factors et le groupe Heller). Les membres de ces groupes ont, dans les divers pays, un actionnaire commun (ex. la First National Bank of Boston pour International Factors);

- celles qui opèrent isolément, mais ont des accords de coopération avec d'autres sociétés d'affacturage indépendantes (ex. Factors Chain International).

Dans certains pays ce sont les organismes officiels d'Assurance-Crédit qui offrent ce type de service.

\subsection{L'Assurance-Crédit et les organismes internationaux}

La majorité des compagnies para-étatiques ou étatiques s'occupant dans le monde de l'Assurance-Crédit se sont regroupées dans l'Union Internationale d'Assureurs de Crédit et des Investissements, communément appelée "Union de Berne ». Les membres travaillent sur la base de "gentlemen agreement» et s'engagent à respecter certaines conditions en matière de couverture du crédit.

C'est au sein de cette Union que fut éditée, en 1953, la règle dite des "cinq ans », selon laquelle la durée de l'assurance ne devrait pas dépasser cette période pour les biens d'équipement lourd.

C'est par contre au sein de l'ICIA que l'on retrouve les principaux assureurs privés; le but recherché, dans ce cas, étant de promouvoir la collaboration internationale sur le plan de l'information, de la surveillance des risques-crédit et la mise au point des principales techniques de souscription.

L'O.C.D.E. s'est également occupée des problèmes de l'Assurance-Crédit: en 1963 s'est constitué un groupe réunissant les représentants des pays membres qui s'est donné pour tâche de neutraliser les distorsions de la concurrence et lutter contre la surenchère des crédits. Pratiquement ce groupe s'est efforcé de fixer, par des accords sectoriels, la durée de crédit maximum applicable à certains types d'équipement. Le premier accord, qui a servi de modèle pour les autres, concernait l'exportation de navires.

Au niveau de la Communauté Européenne existent depuis 1960 deux organismes s'occupant de ces problèmes. Le premier est constitué par un groupe ad hoc, composé de fonctionnaires représentant les pays membres, qui a pour mission de coordonner les politiques nationales d'Assurance-Crédit. Le deuxième, dénommé Comité Technique des Assureurs-Crédit de la $\mathrm{CE}$, a orienté ses travaux dans trois directions:

- recherche d'une politique de crédit commune à l'égard des pays tiers ;

- collaboration entre assureurs pour la garantie des marchés comportant des parts sous-traitées dans la communauté ou les marchés conclus conjointement par les exportateurs des pays membres;

- harmonisation des techniques de l'Assurance-Crédit.

Notons toutefois que les efforts de ces organismes n'ont pas encore pu aboutir à des décisions définitives. C'est ainsi que, devant les difficultés rencontrées pour harmo- 
niser les crédits à l'exportation, la Commission Européenne a récemment proposé de créer une Banque Européenne pour l'Exportation (BEE) dont le triple but serait de :

- faciliter les exportations de biens et de services qui concernent deux Etats membres au moins ;

- harmoniser les crédits à l'exportation accordés par les divers pays membres ;

- pouvoir concurrencer l'Import-Export Bank des Etats-Unis et du Japon.

Mais c'est dans un cadre plus pragmatique que l'on a conclu, récemment, un gentlemen agreement entre les six grandes puissances commerciales pour essayer de limiter la course au crédit et de bien faire la différence entre ce qui pourrait être appelé une aide au développement et des conditions trop avantageuses de vente à crédit.

\section{L'Assurance-Cautionnement à l'étranger}

L'organisme qui délivre ce cautionnement s'engage à honorer un contrat conclu entre deux parties, en cas de défaillance du fournisseur à cause d'incapacité technique ou financière.

Cette forme d'assurance connaît actuellement un développement certain, plus particulièrement dans le domaine des travaux publics, où l'importance croissante des contrats conclus incite les gouvernements et les industriels à se couvrir contre la défaillance éventuelle de l'entreprise à laquelle ils ont confié des travaux.

Les sociétés qui délivrent ces cautionnements sont :

- des assurances, privées ou étatiques;

- des banques, agissant en pool.

La garantie accordée par l'organisme émetteur va très loin : elle peut, en effet, consister non seulement en une prestation en sommes d'argent mais aussi en prestations en nature (ex. engagement d'achever les travaux, donc de rechercher soit un autre entrepreneur, soit de dédommager le maître de l'ouvrage pour les pertes provoquées par la défaillance de l'entrepreneur).

Voici les principaux types de cautionnements accordés :

- Caution provisoire pour concourir («bid bond》) :

l'organisme qui a ouvert l'adjudication est assuré que l'entreprise adjudicataire souscrira le contrat ;

- Caution définitive d'adjudication (《performance bond》): c'est la caution la plus couramment accordée. L'adjudicateur est assuré que la société adjudicataire fournira le travail promis selon les conditions stipulées dans le cahier de charges ;

- Garantie de l'entretien (《maintenance bond》): par la société adjudicataire des installations auxiliaires pendant les premières années d'exercice ;

- Garantie de pénalité ("penalty bond》)

que l'adjudicataire sera éventuellement appelé à payer en cas de retard dans la livraison de l'ouvrage; 


\section{- Garantie de restitution par l'adjudicataire ("advance-payment bond》)}

des montants avancés par l'adjudicateur à l'entrée de l'adjudication. Elle permet notamment aux entreprises d'obtenir de l'adjudicateur le paiement anticipé des retenues de garantie (par exemple, dès l'achèvement des travaux, mais avant leur réception).

Les garanties portant sur les travaux à l'étranger sont souvent exigées à $100 \%$ du montant total de l'adjudication.

Plus récemment, il est devenu assez commun, dans certaines parties du monde, d'exiger de l'adjudicataire une garantie inconditionnelle ("on-demand bond») pour couvrir les avances que l'adjudicateur aurait faites. Cette garantie peut être demandée à tout moment par l'adjudicateur, sans aucune référence aux termes du contrat et sans aucune justification. Quand l'adjudicateur est un organisme public, il est possible de s'assurer contre ce risque, à condition qu'aucune violation du contrat n'ait été commise par l'adjudicataire.

\section{L'Assurance des investissements privés à l'étranger}

\subsection{Introduction}

Jusqu'à la deuxième guerre mondiale les investissements privés à l'étranger prenaient la forme de placements de portefeuille; les fonds se déplaçaient des pays offrant des taux d'intérêt bas vers les pays offrant des rendements supérieurs ${ }^{15}$.

Depuis 1945, par contre, ces opérations ont, le plus souvent, pris l'une des deux formes suivantes :

- création de filiales à l'étranger ;

- ou rachat de firmes étrangères déjà existantes.

C'est ce qu'on appelle les "investissements directs », ce qui implique, de la part de l'investisseur, un contrôle plus strict, ainsi d'ailleurs qu'un apport, technique et financier, plus poussé.

Au surplus, ces opérations ont été caractérisées par divers phénomènes qui ont surpris les observateurs, savoir :

- les investisseurs empruntaient sur le marché local, ou alors l'investissement était financé par le réinvestissement de bénéfices (le capital venant donc de fonds antérieurs) ;

- les investissements directs avaient lieu dans les deux sens (les sociétés américaines, par exemple, investissant en Europe et les sociétés européennes aux Etats-Unis).

Sur le plan théorique, diverses explications ont été proposées pour comprendre ces phénomènes et permettre de prévoir le développement futur de ces investissements.

15 Ainsi l'investissement direct a longtemps été considéré comme un mouvement international de capitaux, au même titre que l'émission de nouveaux titres, l'achat ou la vente de titres en bourse, etc. 
Les principales théories avancées font appel $\mathbf{1 6}$ :

- à la croissance de la firme. Selon cette théorie, les entreprises, en augmentant de dimension, sont naturellement amenées à étendre le cadre de leurs activités en dehors des frontières nationales;

- à la notion de croissance monopolistique. D'après cette thèse, la société qui investit à l'étranger doit dégager un profit supérieur :

- non seulement à celui qu'elle réalise dans son pays d'origine ${ }^{\mathbf{1 7}}$,

- mais aussi à celui des entreprises locales ${ }^{18}$.

On peut résumer paradoxalement cette théorie en disant que, dans un monde utopique de concurrence parfaite, l'investissement direct ne saurait exister. Les imperfections qui faussent la concurrence sont celles, par exemple, résultant d'un progrès technique récent, de l'existence d'économies à l'échelle, etc. Ces imperfections donnent alors un avantage à la société étrangère sur les firmes locales concurrentes ou pouvant exister ${ }^{19}$.

$\mathrm{Si}$, ainsi que nous venons de le voir, l'unanimité n'est pas réalisée sur les causes étant à la base du développement des investissements directs, il en va de même en ce qui concerne les effets de ces investissements dans les pays hôtes.

Ce dernier problème a été étudié dans le contexte du développement des pays du tiers monde. En partant de l'idée que ces pays ont besoin d'un certain montant de capitaux pour leur développement, et constatant que les apports et l'aide publique n'étaient pas suffisants, on a été amené à mettre l'accent sur la nécessité de favoriser les investissements privés, pour combler ce fossé.

\subsection{Les risques relatifs aux investissements privés à l'étranger}

Nous nous intéressons seulement aux risques non commerciaux auxquels sont soumis les investisseurs privés, et parmi ces risques uniquement à ceux qui ne sont pas normalement couverts par les polices traditionnelles de l'assurance incendie.

Autrement dit, nous nous intéressons seulement aux risques politiques ${ }^{20}$, dont voici les principaux :

- risque d'expropriation ou nationalisation, soit ouverte, soit déguisée ${ }^{21}$;

16 Cf. Charles P. Kindleberger: Les Investissements des Etats-Unis dans le Monde. Calman-Lévy, 1971.

17 Pour couvrir les risques et les coâts d'une opération dans un environnement politique, économique et légal différent.

18 Car autrement les entreprises locales, ne courant pas ces risques, auraient des couts défiant toute concurrence.

19 On peut illustrer cette théorie en utilisant la formule simple de capitalisation d'un flux de revenu, $C=I / r$, où $C$ est la valeur du capital, I le flux de revenu que ce capital produit et $r$ le taux de rendement de l'investissement. Alors que les mouvements de capitaux ordinaires s'expliquent par des différences en $r$, les investissements directs correspondent à une différence en I, flux de revenu, entre les entreprises étrangères et les firmes locales.

20 Notons la tendance à élargir le concept de risques politique au-delà des actes des gouvernements pour y inclure également les agissements de terroristes politiques.

21 Selon une publication des Nations Unies il y a eu, dans la période 1960 -millieu 1974, quelque 875 cas de nationalisation dans 62 pays (cf. E/5425/Add. 1). 
- risque de guerre, guerre civile, rébellion, émeutes;

- risques de transfert, couvrant tous les obstacles qui peuvent s'opposer au rapatriement, par l'investisseur, dans son pays, soit du rendement de l'investissement, soit du capital lui-même.

\subsection{Les possibilités de protection des investissements privés}

La protection des investissements est une notion moderne, puisqu'elle date de la première guerre mondiale 22 .

Schématiquement ont peut distinguer deux moyens d'action, d'ailleurs complémentaires, pour essayer de protéger ces opérations :

- le premier, qui a pour effet de viser à prévenir de tels risques, ou, le cas échéant, de les résoudre par la voie juridique (c'est le cas de la BIRD) ${ }^{23}$;

- le deuxième, qui vise à compenser les pertes résultant de la réalisation de ces risques (ce qui constitue l'approche de l'assurance).

Dans le cadre de cette seconde approche on peut se poser la question de savoir quelle est l'importance de l'assurance dans la prise de décision d'investir. réponse :

Une étude réalisée par les services de la BIRD ${ }^{24}$ donne quelques éléments de

- d'après des questionnaires envoyés à des investisseurs, il résulterait que le manque de possibilités de s'assurer aurait conduit, dans certains cas, à la décision définitive de ne pas investir ${ }^{25}$;

- si la motivation de l'investissement est, pour la société, d'être internationale par rapport aux matières premières ou au marché, l'influence de l'assurance semble négligeable quant à la décision d'investir. Par contre elle peut influencer la localisation de l'investissement ;

- si, comme c'est le cas généralement pour l'industrie manufacturière, l'investissement est fait en fonction du profit potentiel, des disponibilités de matières premières et de main-d'œuvre, etc., alors que le climat politique semble instable, la possibilité de s'assurer peut jouer un rôle important.

\subsection{Les systèmes nationaux d'assurance des investissements privés}

Le but recherché par les systèmes nationaux est d'encourager les investissements privés à l'étranger, en accordant aux ressortissants des protections à travers l'assurance.

22 Jusqu'en 1914 on considérait les nationalisations comme possibles à condition d'être précédées d'une indemnisation équitable. Et comme le montant de cette indemnisation était le plus souvent imposé par le pays qui protégeait un ressortissant victime de l'action du gouvernement étranger, cela ne posait pas trop de problèmes (cf. L. Kopelmanas : « La protection des investissements à l'étranger », article paru dans D.P.C.I. de janvier 1975).

23 Mais aussi des divers accords bilatéraux conclus entre pays investisseurs et pays hôtes.

24 Cf. « Multilateral Investment Insurance », BIRD, mars 1962.

25 Encore aurait-il fallu, si elle avait existé, que la garantie offerte corresponde aux besoins exprimés pour que l'investissement soit effectivement réalisé. 
Tous ces systèmes ont des points en commun :

- risques:

les risques couverts sont généralement l'expropriation, la nationalisation ou confiscation ouverte (certains ne couvrent pas l'expropriation déguisée), le risque de guerre, révolution, rébellion et le risque de transfert. Tous excluent également le risque de dévaluation de la monnaie locale;

- type d'investissement :

ne sont couverts que les nouveaux investissements ${ }^{26}$;

- durée de l'investissement:

elle varie de trois à quinze-vingt ans ;

- nationalité de l'investisseur:

tous les systèmes ont prévu des restrictions pour les investissements des entreprises multi-nationales et ne sont disponibles que pour les sociétés qui appartiennent à des nationaux et exercent une activité dans leur pays ;

- les primes sont identiques pour tous les pays hôtes;

Ces systèmes par contre diffèrent sur les points suivants :

- certains ne couvrent que les investissements réalisés dans les pays en voie de développement et d'autres que les investissements réalisés dans les pays ayant conclu des accords bilatéraux pour la protection des investissements ;

- certains insistent sur la nécessité que ces investissements soient favorables pour les pays hôtes et d'autres prennent en considération, avant d'accorder l'assurance, des facteurs tels que la méthode de financement, les rendements prévus (qui ne doivent pas être excessifs), etc.

Financièrement ces systèmes doivent s'équilibrer à long terme : autrement dit, en longue durée, ni bénéfice ni perte.

Parmi les systèmes nationaux il $\mathrm{y}$ en a un qui mérite une attention particulière, à cause de la participation conjointe des autorités nationales et de sociétés d'assurance privées. Il s'agit du système américain.

$\mathrm{Au}$ début des années 70, à la suite d'importantes pertes subies en Amérique du Sud, on a assisté à une tentative de retour progressif de l'organisme public, OPIC, dans le secteur privé. C'est ainsi que, en 1971, l'OPIC concluait un accord valable pour trois ans avec le marché privé londonien, à travers Lloyd's, pour réassurer une partie des risques garantis.

Continuant dans cette voie, l'OPIC a conclu un accord en 1974 avec un groupe d'assurances privées, portant sur la création du Overseas Investment Insurance Group (OIIG). Il s'agit en fait d'un pool, dans lequel l'OPIC participe en tant que membre et envers lequel il agit en qualité de réassureur pour les excès de risque.

26 L'idée est la suivante: on veut encourager les flux de nouveaux capitaux vers les pays en voie de développement. 
L'OIIG couvre les risques d'expropriation et d'inconvertibilité pour environ $2 / 3$ des nouveaux investissements américains (à l'exception des investissements pétroliers) dans quelque 30 pays.

\subsection{Le système privé d'assurance des investissements}

Il n'y a, à l'heure actuelle, qu'un seul système d'assurance privé permettant de couvrir certains risques politiques relatifs aux investissements privés étrangers : il s'agit du système proposé par Investment Insurance International, société créée en 1972, par les Lloyd's de Londres.

Il s'est développé pour répondre aux besoins des investisseurs privés qui ne pouvaient pas s'assurer auprès des systèmes nationaux. Cette assurance, qui était très limitée au début pour permettre d'établir un portefeuille solide, est basée sur un système d'allocation avec des quotas pour :

- les pays recevant l'investissement;

- les pays investisseurs;

- les secteurs industriel couverts.

Dans la mesure du possible on demande à chaque assuré de couvrir la totalité de ses investissements, pour éviter la pré-sélection des risques.

Par rapport aux systèmes nationaux les principales différences sont :

- les primes sont déterminées individuellement pour chaque assuré ;

- la garantie s'applique à tous les investissements et non pas seulement aux nouveaux ;

- la garantie peut s'appliquer à tous les pays et peut être souscrite par toutes les entreprises, y compris les multi-nationales.

Les risques couverts sont:

- expropriation, nationalisation, spoliation ;

- risque de non-convertibilité, mais seulement si elle découle d'une expropriation ;

- possibilité de s'assurer, dans une police séparée, contre des émeutes, grèves et dommages provoqués avec préméditation (《malicious damage »).

Par rapport aux systèmes nationaux sont exclus les risques de guerre et la garantie contre le risque de transfert est plus limitée.

\subsection{Le rôle de la BIRD}

Nous avons fait allusion à la section 3.2 au rôle de la Banque mondiale dans la protection des investissements. Elle a en effet contribué à la création d'un Centre International pour le Règlement des Différends relatifs aux Investissements (CIRDI).

L'objet du Centre est «d'être à la disposition des parties à certains accords internationaux d'investissement, de leur fournir un tribunal assuré auprès duquel ils puissent convenir de soumettre tout différent à venir et de les aider à régler les différends qui ont déjà pu naître ». La juridiction du Centre est fondée sur le consentement des deux parties en cause, l'une doit être un gouvernement (ou un organisme dépendant du gouvernement) et l'autre une personne privée, ressortissant de l'autre Etat partie au litige. 
La portée de cette Convention est assez limitée, car il faut non seulement que les deux Etats l'aient ratifiée, mais encore que, dans le cas d'espèce, les deux parties veuillent bien utiliser la procédure d'arbitrage prévue à cet effet ${ }^{27}$.

On peut noter également que la Banque mondiale avait essayé de créer une agence d'Assurance des Investissements Internationaux, afin de réunir les divers systèmes nationaux en un seul organisme. Mais ce projet, à cause de l'opposition de certains pays membres, n'a jamais abouti.

\section{Quelques lignes de recherche}

Après cette analyse de quelques aspects de l'assurance dans les relations économiques internationales en voici certains qu'il nous semble intéressant d'approfondir.

Il est clair que ces divers aspects devraient être situés dans leur contexte économique général, et qu'une des questions fondamentales qui se pose est bien celle de l'endettement extérieur d'un nombre croissant de pays. Cet endettement peut en effet accroître considérablement les risques: il conviendrait donc de tenir compte de son impact actuel et futur sur la capacité financière des gouvernements, organisations internationales et assureurs d'apporter une garantie de bonne fin aux diverses opérations économiques.

\subsection{Les systèmes d'assurance à l'exportation}

La crise économique actuelle a eu pour effet de mettre en évidence les systèmes d'assurance à l'exportation, considérés essentiels pour maintenir la compétitivité extérieure des industries nationales.

Dans ce contexte les points suivants pourraient être analysés plus en détail.

\section{COMPARAISON DES DIVERS SYSTEMES}

- analyse des principaux systèmes d'assurance privés et publics et de leurs faiblesses ;

- analyse du financement des exportations (en effet pour pouvoir porter un jugement sur les divers systèmes encore faut-il tenir compte des facilités de financement);

- quantification des exportations, selon le type de financement et le type de produits, par exemple :

- matières premières et biens de consommation,

- biens en capital, biens de consommation durables et biens d'équipement légers,

27 Une appréciation du rôle de la BIRD dans le règlement des différends relatifs aux investissements étrangers devrait encore tenir compte de deux actions plus pragmatiques:

- en effet la Banque, à l'occasion des prêts qu'elle est amenée à accorder, est tout naturellement amenée à faire " pression amicale » sur les autorités gouvernementales des pays hôtes pour que le service de la dette extérieure soit régulièrement poursuivi ;

- au surplus le Président de la Banque a souvent engagé son prestige individuel pour favoriser un règlement à l'amiable dans certaines situations embrouillées (par exemple lors de la nationalisation du Canal de Suez).

Il faut signaler aussi que les investissements auxquels le groupe de la Banque participe doivent avoir reçu l'approbation du pays hôte. 
- biens d'équipements lourds, projets clés en main, travaux d'infrastructure ;

- développement de la part des exportations qui est assurée (par type de produit) et évolution des principaux risques couverts (par exemple risques commerciaux par rapport aux risques politiques).

\section{EFFETS DES SYSTEMES D'ASSURANCE A L'EXPORTATION}

- liens entre assurance et financement des exportations ;

- autres effets favorables de l'assurance dans le développement du commerce international (ex. possibilité de se couvrir contre certains risques spécifiques auxquels sont soumis les opérateurs avec l'étranger);

- analyse des subventions pouvant résulter des systèmes d'assurance. Nous avons vu que les systèmes nationaux pouvaient être à l'origine de subventions à l'exportation. Il s'agirait, dans une première phase, de mettre en évidence l'existence et l'étendue de ces subventions, pour ensuite apporter un jugement basé sur la théorie économique en étudiant :

- les effets des subventions à l'exportation, par rapport à une situation de libre échange, sur la production, la consommation, la balance des paiements, le revenu et sa redistribution;

- les effets comparatifs entre les subventions à l'exportation et d'autres obstacles aux échanges internationaux;

- les effets particuliers (par ex. lien entre les crédits à l'exportation et l'aide aux pays en voie de développement);

- les effets de discrimination en fonction du critère de résidence ou nationalité, discrimination entre les divers produits, etc.

\section{LES SYSTEMES D'ASSURANCE}

\section{DANS LES PAYS EN VOIE DE DEVELOPPEMENT}

- ces pays se trouvent parfois pris dans un cercle vicieux : en raison du volume limité des exportations assurables (les exportations de produits primaires se faisant, dans la grande majorité des cas, contre paiement comptant ou en consignation) ils ne peuvent créer des systèmes viables d'Assurance-Crédit à l'exportation. L'absence de ces systèmes, à son tour, décourage l'exportation de nouveaux produits ;

- il faudrait donc mettre en lumière les réalisations déjà existantes dans quelques pays du tiers monde, estimer l'importance de ces systèmes dans le développement des exportations, etc., et les possibilités de coopération entre assureurs du monde industrialisé et des pays en voie de développement.

\section{L'HARMONISATION DES DIVERS SYSTEMES D'ASSURANCE}

- après avoir analysé la diversité des systèmes existants et les effets de cette diversité, il faudrait s'interroger sur les possibilités d'arriver à une harmonisation dans ce secteur, que ce soit au travers des accords internationaux, au travers des systèmes régionaux, etc. ; 
- une analyse de l'œuvre accomplie par la coopération internationale (que ce soit, par exemple, au sein de l'Union de Berne, de l'O.C.D.E., de la Commission Européenne, etc.) permettrait de mieux apprécier les possibilités existant dans ce secteur.

\subsection{Les systèmes d'assurance des investissements privés à l'étranger}

Nous avons vu l'importance croissante des investissements privés et la "Mission 》 qui leur était accordée dans le cadre du développement économique des pays du tiers monde. Dans ce contexte les points suivants nous semblent mériter une attention particulière.

\section{COMPARAISON DES DIVERS SYSTEMES D'ASSURANCE}

- analyse des divers systèmes, publics et privés, existant dans le monde et leurs faiblesses ;

- quantification des investissements directs, en analysant :

- les facteurs qui ont provoqué leur croissance,

- leur évolution passée et prévisible,

- leur répartition géographique ot par secteur d'activité ;

- développement des investissements couverts par l'assurance et principaux risques assurés par pays (ou groupes de pays) et secteurs d'activité ;

- possibilité d'aboutir à une agence internationale d'assurance des investissements internationaux à la lumière des expériences déjà réalisées par les organisations internationales (ex. BIRD).

\section{ROLE DES SYSTEMES D'ASSURANCE DES INVESTISSEMENTS PRIVES}

La question qui se pose est de mieux connaître l'importance de l'assurance (ou l'existence de cette possibilité) dans la prise de décision d'investir. Autrement dit, l'assurance influence-t-elle le montant des investissements existants, oriente-t-elle leur localisation géographique?

\section{LES EFFETS DES INVESTISSEMENTS PRIVES}

Il s'agit là d'un point très controversé, mais qui, après tout, est fondamental ; quels sont les effets de l'investissement privé étranger sur l'économie du pays receveur?

D'un point de vue théorique, il faudrait analyser l'effet sur:

- le marché des facteurs;

- le marché des produits finis;

- les termes de l'échange;

- la balance des paiements.

Cette approche théorique devrait être complétée par une analyse plus pragmatique, qui tiendrait compte des implications politiques et de l'évolution de l'attitude des pays receveurs. 


\subsection{Le rôle de l'assurance privée}

$\mathrm{Ce}$ thème coiffe en quelque sorte les deux premiers.

Il s'agirait de bien mettre en lumière les possibilités et les limites de chaque type d'assurance, publique et privée, et d'analyser en détail les raisons qui, dans certains cas, rendent indispensable actuellement l'intervention de l'assurance publique, pour substituer ou compléter l'assurance privée.

On pense généralement que les gouvernements sont les mieux placés pour discuter, d'égal à égal, en cas de mesures politiques prises par des gouvernements étrangers à l'encontre de leurs ressortissants ou de leurs biens. On peut se demander toutefois si le développement des relations internationales et leur complexité croissante ne vont pas amener progressivement les Etats à hésiter avant de trop s'engager sur ce plan, au risque de voir leur politique étrangère compliquée ou compromise par de telles négociations. Au surplus les sociétés privées peuvent souvent agir, de par leur nature, par des voies plus souples, sans les contraintes politiques ou idéologiques, pour aboutir finalement à des compromis satisfaisants. L'Etat pourrait donc devenir en quelque sorte la dernière voie de recours, si toutes les autres solutions avaient échoué.

Nous pensons que le rôle de l'assurance privée est appelé à se développer également dans le secteur des risques politiques, soit d'une manière indépendante, soit en coopération avec les gouvernements respectifs.

Une autre considération milite en faveur de cette thèse. Nous faisons allusion à l'existence, dans certains pays arabes, d'importants capitaux en quête de placements à long terme. Il n'est pas utopique d'envisager des investissements triangle, dans lesquels les pays détenteurs de capitaux apporteraient leur soutien financier et les pays industrialisés leur technologie. Dans de telles opérations on peut se demander quelle aide les systèmes d'assurance pourraient apporter. Ce problème peut se poser également avec les exportations.

Les assureurs privées peuvent en outre, soit directement, soit indirectement, apporter une aide et une coopération certaine aux pays en voie de développement dans leur recherche de moyens permettant la croissance et la diversification de leurs exportations.

Une dernière remarque: on parle de libéraliser le commerce international des services (aussi bien entre les pays développés qu'entre ceux-ci et les pays du tiers monde dont l'assurance, par sa structure, est l'un des plus importants. Un bon moyen pour préparer le terrain à ces mesures est justement de montrer les effets positifs qu'une plus grande liberté de mouvement de l'assurance, sous toutes ses formes, pourrait avoir. 